\title{
The validity of three snow leopard subspecies: response to Senn et al.
}

\author{
J. E. Janecka ${ }^{1} \cdot$ M. J. Janecka ${ }^{2} \cdot$ K. M. Helgen ${ }^{3} \cdot$ W. J. Murphy ${ }^{4}$
}

Received: 19 October 2017 / Revised: 4 January 2018 / Accepted: 8 January 2018 / Published online: 13 February 2018

(c) The Genetics Society 2018

We recently published the first phylogeographic analysis of snow leopards (Panthera uncia) supporting delineation of three subspecies: P. u. irbis (northern-Mongolia), P. u. uncia (western-Kyrgyzstan, Tajikistan, Pakistan, northwestern India), and $P$. u. uncioides (central-Nepal, Bhutan, China) (Janecka et al. 2017). We analyzed 70 individuals from 21 geographically dispersed sampling sites with 33 nuclear microsatellite loci and 683-bp of mitochondrial DNA (mtDNA). We implemented methods comparable to those used in previous well-accepted studies of felid phylogeography (e.g., Uphyrkina et al. 2001, Luo et al. 2004, Bertola et al. 2015). Within these subspecies, we also identified management units (MU) for which policies should be developed, consistent with the political, economic, and cultural issues relevant to the respective MU. Our taxonomic revision is of great importance to the conservation of snow leopards, and other high-altitude species of Asia.

Our delineation has been criticized by Senn et al. (2017), who claimed that the snow leopard should remain monotypic. This conclusion is neither supported by the results of our phylogeographic study nor consistent with the standards used in the most recent taxonomic revision of Felidae (Kitchener et al. 2017). The genetic evidence amassed in our study was greater than data used to define subspecies for many felids, including margay (Leopardus wiedii), ocelot

J. E. Janecka

janeckaj@duq.edu

1 Department of Biological Sciences, Duquesne University, Pittsburgh, PA, USA

2 Department of Biology, Texas A \& M University, College Station, TX, USA

3 School of Biological Sciences, University of Adelaide, Adelaide, SA, Australia

4 Department of Veterinary Integrative Biosciences, Texas A \& M University, College Station, TX, USA
(Leopardus pardalis), serval (Leptailurus serval), caracal (Caracal caracal), African golden cat (Caracal aurata), jungle cat (Felis chaus), and rusty-spotted cat (Prionailurus rubiginosus) (Kitchener et al. 2017). For three of these species (serval, caracal, and African golden cat), the subspecies were defined solely on biogeographic patterns of sympatric taxa. Among the 26 non-monotypic felids, $78 \%$ of the 77 subspecies recognized by Kitchener et al. (2017) are not morphologically distinct. In fact, for the species above Kitchener et al. defined subspecies that have been accepted by the IUCN Cat Specialist Group using information, which is not even sufficient to delineate MUs when applying the definitions summarized by Senn et al. (2017) in their Table 1 . Therefore, our evidence supporting subspecies delineation of the snow leopard exceeded the minimum criteria used in the recent Felidae taxonomic revision, which Senn et al. reference as having applied "appropriate and consistent guidelines on the appropriate taxonomic criteria for conservation purposes".

Two of our three snow leopard subspecies are in fact supported by Kitchener et al. (2017), who stated that a northern subspecies is "Possibly distinct; molecular study urgently required", based on two independent lines of evidence: morphology and biogeography. Our study provided the third line of evidence (molecular), substantiating a minimum of two subspecies. Further, the range-wide analysis also revealed marked genetic divergence in the southern portion of the range, comparable to that between the northern subspecies, $P$. u. irbis, and the central subspecies, $P$. u. uncioides, justifying the definition of the western subspecies, $P$. u. uncia. This is consistent with a previous assessment by Stroganov (1962), who noted that the snow leopards in parts of Central Asia likely represent a unique subspecies, although the number of specimens he examined was inadequate to make definitive conclusions at the time.

Senn et al. argued the snow leopard remain monotypic using four primary reasons: (1) sampling, (2) mtDNA diversity, (3) admixture, and (4) biogeography. They also 
claimed that we used incorrect nomenclature, when in fact they misinterpreted the relevant taxonomic rules. We address these points below. Although we acknowledge that others may appreciate our analyses of genetic variation in snow leopards without choosing to recognize subspecies boundaries, we reiterate our study does indeed support three subspecies.

(1) Sampling. Senn et al. claimed our study lacked adequate sample sizes. However, the number of individual samples and genetic markers analyzed in our study (77 individuals, 21 locations, 33 microsatellites, 628-bp of mtDNA), is comparable to, and in many cases greater than, the phylogeographic studies used by Kitchener et al. (2017) to redefine or validate subspecies for other felid taxa. These include the leopard (Panthera pardus, 77 individuals, 11 locations, 27 microsatellites, 727-bp of mtDNA, Uphyrkina et al. 2001), ocelot (39 individuals, 16 locations, 0 microsatellites, 444-bp of mtDNA, Eizirik et al. 1998), margay (24 samples, 13 locations, 0 microsatellites, 444-bp of mtDNA, Eizirik et al. 1998), jaguar (Panthera onca, 40 individuals, 20 locations, 29 microsatellites, 715-bp of mtDNA, Eizirik et al. 2001), and jungle cat (55 individuals, 23 locations, 0 microsatellites, 501-bp of mtDNA, Mukherjee et al. 2010). In addition, Senn et al. suggested gaps in our sampling were substantial enough to preclude subspecies delimitation, particularly between northwestern India and Nepal $(\sim 1100 \mathrm{~km})$. Close examination of the subspecies boundaries defined by Kitchener et al. (2017) reveal many were based on studies with even larger sampling gaps. For example, the three margay subspecies were defined solely on one phylogeographic study (Eizirik et al. 1998) with a sampling gap of $\sim 2000 \mathrm{~km}$ between two of them. This study also used $\sim 1 / 3$ of our sample size, $\sim 2 / 3$ of our number of locations, and only three samples from one of the subspecies (i.e., L. w. vigens). In the case of the more extensively studied lion, Panthera leo, there was a $\sim 900 \mathrm{~km}$ sampling gap (Bertola et al. 2016) spanning the subspecies boundary in one of the studies Kitchener et al. (2017) used to demarcate P. l. leo and P. l. melanochaita. Therefore, our snow leopard subspecies delineation was based on larger sampling of individuals across a wider distribution than the minimum used by other studies cited as evidence in the recent taxonomic revision of Felidae (Kitchener et al. 2017). We do acknowledge additional sampling may influence phylogeographic patterns, however, the effects are not easily predicted. Although further analyses will surely provide additional insights, this does not preclude revising the snow leopard taxonomic classification using the most recent data available.

(2) mtDNA diversity. Senn et al. claimed that we cannot delineate subspecies because we found no variation in 638bp of mtDNA. We disagree, and argue that this view places a disproportionately large weight on mtDNA, a single cytoplasmic linkage group, relative to 33 unlinked microsatellite loci. MtDNA also has several unique properties: (1) it is inherited only through females, (2) its effective population size is $1 / 4$ of the nuclear genome, (3) it lacks recombination, and (4) it is located in an organelle, each of these having several notable ramifications (reviewed in Toews and Brelsford 2012). First, mtDNA will show lower variation subsequent to a bottleneck than nuclear markers (e.g., Culver et al. 2000). Second, genetic drift will act to dissociate mtDNA from species-level processes (e.g., Roca et al. 2005). Third, male-biased dispersal and hybridization will largely be undetected in an mtDNA analysis in the absence of nuclear DNA information, whereas ancient maternal hybridization may remain despite nuclear divergence (e.g., Li et al. 2016a). For these reasons, mtDNA lineages and phylogeographic patterns can be in direct conflict with the remainder of the genome (Avise 2000, Toews and Brelsford 2012). However, the discordance of a single molecular marker is not necessarily a shortcoming of only mtDNA, as any locus can be incongruent with the true genealogical history of a species (Avise 2000). A single locus, particularly mtDNA, should not be the primary criterion for accepting or rejecting a new taxonomic classification. In the case of the snow leopard, we argue that the overwhelming evidence provided by 33 independent nuclear loci outweighs data from short mtDNA segments, and we therefore delineated subspecies despite lack of mtDNA divergence.

(3) Genetic structure and admixture. Senn et al. suggested that because there is admixture between the three primary clusters they do not constitute separate subspecies, and instead reveal only population-level structure. The amount of gene flow among populations is one of the primary parameters that determines admixture, evolutionary trajectory, and speciation (Avise 2000). Higher levels of gene flow among subpopulations will cause them to function as a single unit, and if they are isolated from other populations, then they will evolve independently as a metapopulation and given enough time may diverge into evolutionary significant units, subspecies, and ultimately species. However, this divergence can occur even in the presence of admixture (Avise 2000, Li et al. 2016a). Recent whole-genome analyses have recognized that admixture is far more common than previously appreciated, particularly within Felidae (Li et al. 2016a). Many of the felid subspecies have experienced admixture. This process was even reflected in the working subspecies definition provided by Kitchener et al. (2017), which stated "gene flow is expected between subspecies". For example, extensive nuclear and mtDNA admixture was observed between the African lion subspecies $P$. $l$. leo and $P$. $l$. melanochaita recognized by Kitchener et al. (2017) (Bertola et al. 2015,2016,). In our Bayesian structure analysis that modeled genetic clusters 
corresponding to the number of subspecies $(K=3)$ only 5 out of the 70 individuals had mixed ancestry $(Q \leq 0.80)$. All of these were from Kyrgyzstan and still had most of their ancestry (69-75\%) attributed to the appropriate P. u. uncia subspecies (see Figure 2 in Janecka et al. 2017). In contrast, a study of lions by Bertola et al. (2015) performed similar Bayesian modeling of genetic clusters including runs with the same number $(K=2)$ as the subspecies defined by Kitchener et al. (2017). All of the lions from eastern and southern Africa that Kitchener et al. delineated as $P$. $l$. melanochaita were assigned to the same genetic cluster as the western P. l. leo (see Figure 2 in Bertola et al. 2015). In addition, 31 of the 100 lions from Africa showed mixed ancestry $(\mathrm{Q} \sim 0.80)$. We therefore conclude the patterns of admixture in snow leopards do not preclude our proposed taxonomy.

(4) Biogeography. Senn et al. incorrectly claimed that our delineation of three subspecies is not supported by biogeographic evidence. The northern $P$. u. irbis occurs in the Altai and Gobi-Altai Mountains region. To the south, the Gobi Desert forms a natural barrier from the Tibetan Plateau ( $P . u$. uncioides) and to the southwest, the Dzungarian Basin forms a barrier from the Tian Shan Mountains (P. u. uncia). Kitchener et al. (2017) listed "biogeography" as "good evidence" in support of the northern subspecies being "Possibly Distinct". Riordan et al. (2015) constructed a model showing connectivity is nonexistent in the Gobi Desert, and has among the lowest estimates in the Dzungarian Basin (see Figure 2 in Riordan et al. 2015). Riordan et al. (2015) even stated that despite occasional reported sightings of snow leopard in these desert areas "this is still considered the least suitable of the habitat types with the highest barrier to movement". Further, the widely accepted snow leopard distribution map does not show resident populations in the vast majority of the Gobi Desert or Dzungarian Basin (IUCN 2017). Taken together, existing data indicate that major biogeographic barriers do exist, and are consistent with at least two of the three proposed subspecies in Janecka et al. (2017).

Senn et al. also failed to recognize the biogeographic distinction of the Tibetan Plateau/Himalaya and the PamirKarakorum regions. The western boundary of the Tibetan Plateau is the eastern end of the Karakorum (Baud 1989). These regions harbor distinct faunal communities. Of the mammals on the Tibetan Plateau, 23.6\% are endemic (Zheng et al. 1981). Among species with habitat preferences similar to snow leopards, markhor (Capra falconeri) and urial (Ovis orientalis) occur primarily in the PamirKarakorum (IUCN 2017). In contrast, blue sheep (Pseudois nayaur), Himalayan serow (Capricornis thar), Himalayan thar (Hemitragus jemlahicus), Mountain goral (Naemorhedus goral), and takin (Budorcas taxicolor) are found only in the Tibetan Plateau or Himalaya (IUCN
2017). The southern portion of the ibex (Capra sibirica) range is primarily in Pamir-Karakorum, with a minor extension into the very western corner of the Tibetan Plateau/Himalaya (IUCN 2017). The argali, which occurs in both areas, is divided into two allopatric subspecies conforming to the two regions (O. a. hodgsoni vs. $O$. a. polii, Wilson and Reeder 2005). These distributions reveal an important yet underappreciated biogeographic boundary between Pamir-Karakorum and Tibetan Plateau/Himalaya.

In addition, Senn et al. claimed the Pamir Knot (Burrard and Hayden 1907) is recognized as a "major biogeographical link", and this precludes demarcation of two subspecies. This is incorrect for several reasons. First, this region is among the least studied areas of the World and there is no published work that we are aware of that directly tested this hypothesis. Senn et al. do not provide any reference for their statement. Second, there have been no studies to date that have directly examined snow leopard dispersal through this area. Finally, biogeographic boundaries can be present despite contemporary connectivity. One illustrative example is the bobcat (Lynx rufus): a large portion of the boundary between the western L. r. fasciatus and eastern $L$. r. rufus subspecies defined in Kitchener et al. (2017) is formed by the Great Plains, a region which today harbors continuous bobcat populations "with no obvious physical barrier to gene flow" (Reding et al. 2012). The two bobcat subspecies are hypothesized to have originated in disjunct climate refugia during the Pleistocene, and this mechanism is presented by Kitchener et al. (2017) as the main rationale underlying their taxonomic revision. Yet, Senn et al. ignore the potential for similar paleoecological processes influencing the snow leopard.

Our phylogeographic analysis showed much lower connectivity between the Pamir-Karakorum and Tibetan Plateau/Himalaya then postulated in Senn et al. and modeled in Riordan et al (2015), as indicated by the lower admixture in this region compared with the northwest. The Riordan et al. model did not use direct observations but was instead based on 8000 random points plotted within predicted snow leopard range, with only three habitat categories-not surprisingly it over estimated connectivity. In fact, their landscape map showed low resistance far outside of the snow leopard range, such as the coastal region of Pakistan and southeastern China (see Figure 1 in Riordan et al. 2015). A more recent habitat model constructed by Li et al. (2016b) based on 2111 verified snow leopard observations, and more reasonable habitat parameters, indicated reduced connectivity between Pamir-Karakorum and Tibetan Plateau/Himalaya and supported three climate refugia of stable habitat blocks $>50,000 \mathrm{~km}^{2}$, concordant with our phylogeographic analysis and three snow leopard subspecies.

Therefore, the three subspecies proposed in Janecka et al. (2017) are indeed supported by the biogeography of Asia. 
The lines drawn in Figure S6 (Janecka et al. 2017) were used to generate preliminary population size estimates for each subspecies, and we clearly stated more research is needed to demarcate the boundaries between them and validate population assignments.

(5) Taxonomic nomenclature. Senn et al. claimed that our proposed taxonomic nomenclature is incorrect because the name uncioides is a nomen nudum and thus unavailable, and also that the name irbis, proposed as a replacement name for uncia, must be regarded as synonymous with uncia. In the first case, Senn et al. were incorrect about the standing of the name uncioides; and in the second case, we further explain the allocation of the names uncia and irbis using an argument other authors have already employed in past taxonomic arrangements.

The name uncioides was first attributed to Hodgson in a paper published by Horsfield; the name was thus made available by, and takes authorship from, Horsfield. The cursory description demonstrates that Hodgson had a series of syntype specimens available to study, and offers brief comparison with other snow leopard specimens. Like all or most previous authors, we regard uncioides as an available name, as for various other names that Horsfield and others (such as Gray, e.g., Mustela strigidorsa Gray 1853) similarly first erected via attributions to Hodgson. Senn et al. claim that uncioides is a nomen nudum specifically because Horsfield did not designate a type specimen, but this is a misunderstanding in two ways. First, as noted above, a syntype series was available at the time of description; we do not know if these specimens remain extant in any museum collection, but that does not affect the standing of the name per se. Second, the ICZN only requires explicit fixation of name-bearing types for new species-group names proposed after the year 1999 (ICZN 1999: Article 16.4), and this criterion obviously does not apply to the name uncioides, which was published in 1855.

Considerable confusion has always existed about the type locality for the name uncia. The original description by Schreber in 1775 was based on the account of Buffon (from 1761), who figured an unmistakable picture of a snow leopard skin and stated that the animal occurred in Persia. Schreber identified the range of uncia as "Barbarey, Persien, Ostindien, and China"; this obviously involved confusion with the distributions of other species, probably including both the leopard Panthera pardus and the cheetah Acinonyx jubatus. With regard to its possible occurrence in "Persia" (i.e., modern Iran), as mentioned by Buffon and others, Ognev (1935) accepted its historical occurrence in Iran along the Turkmenistan border in the Kopet Dag Mountains, and fixed the type locality as the "southern slopes of these mountains, adjacent to Iran". This attribution is likely incorrect, and probably reflects confusion with Panthera pardus. Nevertheless, the historical and current range of snow leopards includes other countries that border Iran (Afghanistan, Pakistan) and 18th century usage of the term "Persia" by authors such as Buffon and Schreber are best regarded as a loose indicator of the western region of snow leopard occurrence.

Ehrenberg (1830) originally proposed the name irbis as a replacement name for uncia, but in doing so, had a specimen available from the Altai Mountains, making this name available for northern populations of the snow leopard. Pocock (1930) found it convenient to consider irbis and uncia as synonymous, even though it seems unlikely Buffon's skin (the type of uncia) came from somewhere as remote as the Altai Mountains in the mid-1700s. Further, Pocock explicitly acknowledged the possibility that these names might require reassessment if subspecific variation was detected in the snow leopard.

In summary, two previously published alternative interpretations exist for the type locality of Panthera uncia. The first is to accept that Buffon's original specimen likely came from the broadest vicinity of "Persia", as originally stated, i.e., in Central Asia from within the western part of snow leopard the range (Ognev 1935). The second is to accept opinions that assume, out of convenience, that Buffon's specimen came from the Altai Mountains (Pocock 1930). We have chosen the first interpretation. In our three subspecies model, this identifies $P$. u. uncia as the name for the western subspecies and $P . u$. irbis as the earliest name applied to the northern subspecies. Finally, as demonstrated above, $P$. $u$. uncioides remains the earliest available name applied to the central, Himalayan subspecies.

In summary, we disagree with the opinion of Senn et al. (2017) that the snow leopard should remain monotypic. Their arguments are flawed and inconsistent with the recent taxonomic revision of Felidae, which was spearheaded by one of their co-authors (i.e., A. Kitchener). We reiterate our phylogeographic analysis supports three subspecies based on genetic structure and biogeography, and is consistent with previous authors that suggested there are likely multiple subspecies. Further, our proposed names do indeed use the correct taxonomic nomenclature. Given the snow leopard has been recently delisted by the IUCN from Endangered to Vulnerable, our taxonomic revision is an important framework for independently assessing the status of this iconic species within different biogeographic regions. This can be done whether the IUCN and other organizations accept our proposed subspecies, or decide to treat the genetic clusters we identified as MUs. We encourage additional studies that examine our proposed taxonomic revision, however, conclusions should be based on additional data instead of arbitrarily applying more stringent criteria to snow leopards than used for other felids. 


\section{Compliance with ethical standards}

Conflict of interest The authors declare that they have no conflict of interest.

\section{References}

Avise JC (2000) Phylogeography: the history and formation of species. Harvard University Press, Cambridge, MA, USA.

Baud A (1989) The western end of the Tibetan Plateau. In: Sengor AMC (ed). Tectonic Evolution of theTethyan Region. Kluwer Academic Publishers, Dordrecht, Netherlands, pp 505-506.

Bertola LD, Tensen L, van Hooft P et al. (2015) Autosomal and mtDNA markers affirm the distinctiveness of lions in West and Central Africa. Plos ONE 11:e0137975.

Bertola LD, Jongbloed H, van der Gaag KJ et al. (2016) Phylogeographic patterns in Africa and high resolution delineations of genetic clades in the lion (Pathera leo). Sci Rep 6:1-11.

Burrard SG, Hayden HH (1907) A sketch of the geography and geology of the Himalaya Mountains and Tibet. Superintendent Government Printing, Calcutta, India.

Culver M, Johnson WE, Pecon-Slattery J, O'Brien SJ (2000) Genomic ancestry of the American puma (Puma concolor). J Hered 91:186-197.

Ehrenberg MCC (1830) Observations et donées nouvelles sur le tigre $\mathrm{du}$ nord et la panthère du nord, recueillies dans le voyage de Sibérie fait par M. A. de Humbold, en l'année 1829. Ann Des Scences Nat 21:387-412.

Eizirik E, Bonatto SL, Johnson WE, Crawshaw PG, Vie JC, Brousset DM, O’Brien SJ, Salzano FM (1998) Phylogeographic patterns and evolution of the mitochondrial DNA control region in two neotropical cats (Mammalia, Felidae). J Mol Evol 47:613-624.

Eizirik E, Kim J, Menotti-Raymond M, Crawshaw PG, O'Brien SJ, Johnson WE (2001) Phylogeography, population history and conservation genetics of jaguars (Panthera onca, Mammalia, Felidae). Mol Ecol 10:65-79.

Gray JE (1853) Observations on some rare Indian Animals. Proc Zool Soc Lond 1853:190-192.

Horsfield T (1855) Brief notices of several new or little-known species of Mammalia, lately discovered and collected in Nepal, by Brian Houghton Hodgson. Ann Mag Nat Hist 16:101-114.

ICZN (1999) The Constitution of the International Commission on Zoological Nomenclature. The International Trust for Zoological Nomenclature, London.

IUCN. (2017). The International Union for Conservation of Nature Red List of Threatened Species. http://www.iucnredlist.org. Distribution Maps Accessed 17 October 2017.

Janecka JE, Zhang YQ, Li DQ et al. (2017) Range-wide snow leopard phylogeography supports three subspecies. J Hered 108:597-607.
Kitchener AC, Breitenmoser-Wursten Ch., Eizirik E, et al. (2017) A revised taxonomy of the Felidae. Cat News Special Issue 11: $1-80$.

Li G, Davis BW, Eizirik E, Murphy WJ (2016a) Phylogenomic evidence for ancient hybridization in the genomes of living cats (Felidae). Genome Res 26:1-11.

Li J, McCarthy TM, Wang H, Weckworth B, Schaller GB, Mishra C, Lua Z, Beissinger SR (2016b) Climate refugia of snow leopards in High Asia. Biol Conserv 203:188-196.

Luo S, Kim J, Johnson W et al. (2004) Phylogeography and genetic ancestry of tigers (Panthera tigris). Plos Biol 2:e442

Mukherjee S, Krishnan A, Tamma K, Home C, Navya R, Joseph S, Das A, Ramakrishnan U (2010) Ecology driving genetic variation: A comparative phylogepgraphy of jungle cat (Felis chaus) and leopard cat (Prionailurus bengalensis) in India. Plos ONE 5:1-16. e13724.

Ognev SI (1935) Zveri SSSR I priležaščich stran. Mammals of USSR and adjacent countries. V. III. Carnivores. Israel Program Science Translations, Jerusalem.

Pocock RI (1930) The panthers and ounces of Asia. Part II. J Bombay Nat Hist Soc 34:307-336

Reding DM, Bronikowski AM, Johnson WE, Clark WR (2012) Pleistocene and ecological effects on continental-scale genetic differentiation in the bobcat (Lynx rufus). Mol Ecol 12:3078-3093.

Riordan P, Cushman S, Mallon D, Shi K, Hughes J (2015) Predicting global population connectivity and targeting conservation action for snow leopard across its range. Ecography 38:1-8.

Roca AL, Georgiadis N, O'Brien SJ (2005) Cytonuclear genomic dissociation in African elephant species. Nat Genet 37:96-100.

Schreber, J.C.D. von. (1775) Felis uncia. Die Säugthiere in Abbildungen nach de natur. 1774-1855. Tafeln 100.

Senn, H, Murray-Dickson, G, Kitchener, AC, Riordan, P, Mallon, D (2017) RE: Response to Range-Wide Snow Leopard Phylogeography Supports Three Subspecies. Heredity https://doi.org/10. 1038/s41437-017-0015-4.

Stroganov SU (1962) Carnivorous mammals of Siberia. Israel Program Science, Jerusalem, Israel.

Toews DP, Brelsford A (2012) The biogeography of mitochondrial and nuclear discordance in animals. Mol Ecol 21:3907-3930.

Uphyrkina O, Johnson WE, Quigley H, Miquelle D, Marker L, Bush M, O'Brien SJ (2001) Phylogenetics, genome diversity and origin of modern leopard, Panthera pardus. Mol Ecol 10:2617-1633.

Wilson DE, Reeder DM (2005) Mammal Species of the World: A Taxonomic and Geographic Reference, 3rd edn. Johns Hopkins University Press, Baltimore, MD, USA.

Zheng ZX, Feng ZJ, Zhang RZ et al. (1981) On the land-vertebrate fauna of Qinghai-Xizang Plateau with considerations concerning its history of transformation. Geological and ecological studies of Qinghai-Xizang Plateau. Science Press, Beijing, China, pp 975-987. 